\title{
Association of Angiogenic Cells in the Tumour Microenvironment and the Circulating Matured Endothelial Cells in Astrocytic Glioma
}

\author{
Priscilla Das $^{a *}$, Nyi Nyi Naing ${ }^{\mathrm{b}}$, Nadiah Wan-Arfah ${ }^{\mathrm{c}}, \mathrm{KON}$ Noorjan $^{\mathrm{d}}$, Yee Cheng Kueh ${ }^{\mathrm{e}}$, Kantha Rasalingam ${ }^{f}$ \\ ${ }^{a}$ Unit of Biostatistics \& Research Methodology, School of Medical Sciences Universiti Sains Malaysia \\ Faculty of Health Sciences, Asia Metropolitan University \\ ${ }^{b}$ Institute for Community (Health) Development (i-CODE), Universiti Sultan Zainal Abidin \\ 'Institute for Community (Health) Development (i-CODE), Universiti Sultan Zainal Abidin \\ ${ }^{d}$ Departmentof Psychiatry, Faculty of Medicine and Health Sciences, Universiti Putra Malaysia \\ ${ }^{e}$ Unit of Biostatistics \& Research Methodology, Department of Psychiatry, School of Medical Sciences \\ Universiti Sains Malaysia \\ ${ }^{f}$ Department of Neuroscience, Hospital Kuala Lumpur
}

\section{ABSTRACT}

Introduction: Astrocytic gliomas are the most common and lethal intracranial brain tumours and rely on angiogenesis for the tumour development. Endothelial progenitor cells (EPCs) contribute to the angiogenesis of glioma tumour. Objectives: The study aimed to investigate the matured circulating endothelial cells population in the peripheral blood mononuclear cells (PBMCs) and its associations with tissue resident angiogenic cells in astrocytic glioma patients. Methods: A total of 22 astrocytic glioma patients were recruited from Hospital Universiti Sains Malaysia. Tumour were sliced and stained with $\mathrm{CD}_{133^{+}}$and VEGFA ${ }^{+}$ for angiogenic cells $(n=22)$. The circulating (CD133-/VEGFR2 + ) matured endothelial cells in PBMCs $(n=22)$ were quantified using FACS. The paired t-test and Pearson correlation test were used for the data analysis. Results: The angiogenic cells in brain tumour tissue were significantly higher compared to adjacent normal brain tissue (median $1.07 \pm 0.96 \%$ vs. median $0.69 \pm 0.68 \%$; Wilcoxon signed rank test $Z=-3.100$; $\mathrm{p}=0.002$ ). Positive correlation was found between the angiogenic cells of brain tumour tissue and adjacent normal brain tissue (Spearman's rho correlation test, $r=0.56 ; p=0.007$ ). Significant positive correlation was found between matured endothelial cells in peripheral circulating systems and angiogenic cells in tumour of astrocytic glioma patients (Pearson correlation test, $r=0.60, p=0.003$ ). Conclusion:The findings of the study give support to the possible roles of EPCs in astrocytic glioma patients. Thus targeting tissue resident angiogenic cells and matured circulating endothelial cells by antiangiogenic treatment might be useful to prevent the tumour growth.

KEYWORDS: Angiogenic Cells, Circulating Matured Endothelial Cells, Astrocytic Glioma INTRODUCTION

Astrocytic glioma is the most common primary brain tumour which accounts for two third of all tumours

\section{Corresponding author:}

Priscilla Das

1.Unit of Biostatistics \& Research Methodology, School of Medical

Sciences Universiti Sains Malaysia 16150 Kubang Kerian, Kelantan

2. Faculty of Health Sciences, Asia Metropolitan University, G-8, Jalan Kemacahaya 11, Taman Kemacahaya, Batu 9, 43200 Cheras, Selangor Tel no: 010-2070314 Email: daspriscilla@gmail.com of glial origin. ${ }^{1}$ According to WHO classification of central nervous system tumours, astrocytomas are typically classified as pilocytic (grade I - less aggressive), diffuse (grade II), anaplastic (grade III), and glioblastoma multiforme (GBM) (grade IV- most aggressive) in order of increasing anaplasia. ${ }^{2}$

When tumours reach a certain size, the increased requirement for oxygen and nutrients lead to the growth of new blood vessels. Many recent studies have demonstrated the existence of additional angiogenic and vasculogenic mechanisms that are associated with tumour growth. Intussusceptive angiogenesis, vessel co-option, vasculogenic 
The EPCs are the recruited from the bone marrow to form new blood vessels. ${ }^{6-9}$ However the newly formed vessels were less disorganised, tortuous, dilated, leaky and hemorrhagic. ${ }^{6,10-12}$ The previous studies have been reported that the bone-marrow derived EPCs to the site of tumour induced abnormal differentiation of these cells into matured endothelial cells which then incorporate into a subset of sprouting tumour neovessels..$^{6-9}$ Thus the EPCs have ability to differentiate into matured endothelial cells which plays important role in vessel formation and contribute both structurally and functionally to tumour neoangiogenesis. This statement further supported by Daniel et al who defines the EPCs cells surface expression of VEcadherin,VEGFR2, CD31 low, Endoglin, and Prominin I/AC133 differentiate into matured endothelial cells. ${ }^{7}$

The ability of circulating endothelial cells homing to the sites of tumour showed their capacity to be used as a biomarker for the diagnosis and monitoring tumour aggressiveness as well as measuring tumour angiogenicity. ${ }^{7,10,11}$

The development of anti-angiogenic anti-vascular endothelial growth factor for the treatment of astrocytic glioma tumour progression has been modest and it has been reported to be associated with various adverse effects such as delayed wound healing, hypertension, thrombotic events and congestive heart failure. ${ }^{13}$ The development and optimization of biomarkers to measure angiogenesis in tumours, such as measurement of the numbers of circulating EPCs, would potentially help the physicians and surgeons to identify patients that are responding to, or failing the anti-angiogenic therapy.

Therefore, there is a need for the improvement and development of current therapeutic treatment in the management of astrocytic glioma, as none of the available treatments have been shown to be sufficient to either prevent or treat the disease effectively in astroglioma patients. In the current study, it was generally speculated that bone marrow derived EPCs (circulating and tissue resident EPCs) have a significant effect on astrogliomas pathophysiology. Most previous studies only focused either on the circulating EPCs or tissue specific EPCs independently. Unfortunately, none had studied the association between circulating and tissue specific
EPCs in astrocytic gliomas. Hence, this study is specifically designed to fill the gap.

Until recently, few treatment options were available for high grade astrocytic gliomas. However, despite significant advances in surgery, radiotherapy, and chemotherapy over the past 3 decades, the median patient survival remains $<2$ years from lesion diagnosis. ${ }^{14-16}$ Although extensive works have been conducted, the treatment options for these tumours remain limited. ${ }^{2}$

Therefore we are intend to quantify and find the associations between matured circulating endothelial cells population in the peripheral blood mononuclear cells (PBMCs) and its associations with tissue resident angiogenic cells including tumour and normal adjacent brain in astrocytic glioma patients. Hence, our general aim is to add valuables new information that may be useful in improving the efficiency of anti-angiogenic therapy for astroglioma patients.

\section{METHOD}

\section{Patient Population}

A total of 22 astrocytic glioma patients (WHO grade I to IV) from the Hospital of Universiti Sains Malaysia (HUSM) that were enrolled consecutively over a 3 year period (2012-2014) were recruited in the study. Patients identified by radiological appearance of astrocytic gliomas with subsequent histological confirmation. Only cases that are unruptured and do not undergo pre-surgical embolization were selected in the study. The Human Research Ethics Committee, Universiti Sains Malaysia (FWA Reg No: 00007718; IRB Reg. No: 00004494) has approved the study.

\section{Blood Sampling}

The peripheral venous blood was obtained from each patient during surgery for the analyses of matured circulating endothelial cells. At the time of surgery the peripheral venous blood $(25 \mathrm{ml})$ was collected in EDTA tube via venipuncture from each patient. To minimize contamination with endothelial cells from the puncture wound of the vascular wall, the initial $5 \mathrm{ml}$ blood samples drawn were discarded. ${ }^{16}$ After collection the blood samples were processed within an hour. 


\section{Flowcytometry Studies}

The circulating matured endothelial cells in peripheral blood are quantitatively determined by using FACSCanto II flow cytometer (Becton Dickinson, USA). The peripheral mononuclear cells (PBMCs) were prepared by gradient centrifugation using Ficoll-Hypaque technique. About $\left(10^{6}\right.$ cells) of PBMC cells were pippetted in separate $1.5 \mathrm{ml}$ centrifuge tube and added with $10 \mu \mathrm{l}$ of FcRblocking reagent for 10 minutes to inhibit any nonspecific bindings. Next the cells were incubated with $10 \mu \mathrm{l}$ of phycoerythrin-conjugated $(\mathrm{PE})$ antihuman CD133 monoclonal antibody (mAb) (Miltenyi Biotech), 10 $\mu$ l of allophycocyanin-conjugated (APC) anti-human VEGFR-2 mAb (Miltenyi Biotech) and FITC annexin (Miltenyi Biotech) for 30 minutes at $4^{\circ} \mathrm{C}$. As a measurement of negative controls the PE, APC- and FITC- isotype-matched IgG1 (Miltenyi Biotech) antibodies were used. The cells then washed 3 times with $1 \mathrm{X}$ Phosphate buffered saline (PBS) and re-suspended in $400 \mu$ l of FACS solution. The FLowJo_V10 software was used to analyse the data. A minimum of 50000 events were collected and each probe were performed in duplicate. The percentage of circulating matured endothelial cells in peripheral blood was determined by $2 \mathrm{D}$ sidescatter/fluorescence dot-plot analysis. Matured endothelial cells were expressed as percentage of total PBMCs in each patient. ${ }^{11}$

\section{Tissue sampling}

Brain tumour microsurgical specimens from the same 22 astrocytic glioma patients were obtained from each patient after the surgery for the analyses of tissue resident matured endothelial cells. Tissue biopsy were fixed in 10\% paraformaldehyde and analysed by immunofluorescence microscopy.

\section{Immunofluorescence Microscopy}

The total percentage of matured endothelial cells in the tumour specimens was characterized using immunofluorescence microscopy method. Tissue biopsy were that fixed in paraformaldehyde $10 \%$ for 48 hours were cut into 3 section with an interval of $6.5 \mathrm{~mm}$ and $4 \mathrm{~mm}$ thickness for brain tumour and normal adjacent brain. The tissue were processed in tissue processor and embedded in wax paraffin block. Tissue blocks were cut in a thickness of $3 \mu \mathrm{m}$ followed by 8 serial dissections. First, the tissue were deparaffinized by using two changes of xylene, followed by xylene 1:1 with ethanol and rehydration with two changes of absolute, $95 \%$ and 70\% ethanol for 3 minutes each. Tissue then was rinsed with running cold tab water and enough drops of $0.1 \%$ Triton $x-100 /$ PBS was added for 10 minutes. Next washed with 1X PBS for 3 times and added with PBS/BSA $0.5 \%$ for 5 minutes.

The tissue sections were stained with Phycoerythrinconjugated (PE) anti-human CD133 (Miltenyi Biotech) and Fluorescein Isothiocyanate (FITC) anti-human VEGFA (Bioss). After overnight incubation at $4^{\circ} \mathrm{C}$ in a dark humid chamber, the tissue were washed with 1X PBS for 3 times and counterstaining were performed with 4',6-diamidino-2-phenylindole (DAPI) for 30 minutes. The slides then washed with 1X PBS for 3 times and mounted in mounting medium and then analysed using BX41 Leica microscopy. The images were captured and merged to see the co localization of the markers.

Excitation in the ultraviolet $(330-385 \mathrm{~nm})$, blue $(460-$ $490 \mathrm{~nm})$ and green $(510-550 \mathrm{~nm})$ are used in this BX41 Olympus microscope. The ultraviolet filter used to identify the cell nuclei stained bluewith DAPI, the blue filter used to detect the FITC-VEGFR2+ marker that is reflected in green and the green filter for PECD133 marker in red. The microscope has magnifications of 20, 40, 100 and 200 power and both the reflected light and sample fluorescence are viewed through and recorded at the microscope. A Nikon Coolpix 5.1 megapixal camera as well as a USB camera with image capture software are used to record the images. ${ }^{17}$

The images were captured and merged to see the expression of the markers. The percentages of tissue resident EPCs were determined in $13.78 \mathrm{~mm}^{2}$ of the tumour tissue area per patient and $13.78 \mathrm{~mm}^{2}$ for adjacent normal brain per patient in immunofluorescence microscopy. A total of 24 fields image were captured for brain tumour sample and another 24 fields for adjacent normal brain per patient. Therefore the total area assessed for 22 patients for both tumour and adjacent normal brain were $606.50 \mathrm{~mm}^{2}$ for tissue resident EPCs. The images were captured at 200X magnification. The counts were expressed as the average of all fields examined. The percentages of EPCs in the tissues were analysed using Image J software version $1.45 \mathrm{~s}$. About $13784 \mathrm{~mm}^{2}$ of the tissue area for each sample of brain tumour and adjacent tissue were assessed. 


\section{Statistical Analysis}

The data presented as median \pm SDs. The paired $t$ tests, Pearson correlation test and Spearman's rho correlation test were performed. The statistical significance was determined at $p<0.05$. SPSS software version 20.0 was used for all the analyses.

\section{Results}

\section{Participant Characteristics}

Twenty two patients with astrocytic glioma were enrolled in this study including (WHO grade $I V, n=9$, grade III, $n=8$, grade $I I, n=2$, grade $I$, $\mathrm{n}=3$ ). The diagnosis of astrocytic glioma patient's that were included in the study were pilocytic astrocytoma, diffuse fibrillary astrocytoma, diffuseastrocytoma, anaplastic astrocytoma, anaplastic oligodendroglioma, anaplastic ependymoma, anaplastic gemistocytic astrocytoma, glioblastoma with oligodendroglioma component, gliosarcoma and glioblastoma multiformae. Summary of participant characteristics are displayed in Table 1.

Table 1: Socio-demographic and clinical characteristics of respondents

\begin{tabular}{lr}
\hline Characteristics & $\mathrm{n}(\%)$ \\
\hline Age & \\
$\quad \leq 40$ years & $8(40.0)$ \\
$\quad>40$ years & $14(60.0)$ \\
Gender & \\
$\quad$ Male & $16(70.0)$ \\
Female & $6(30.0)$ \\
Astrocytic glioma diagnosis & \\
$\quad \begin{array}{l}\text { Glioblastoma multiformae WHO } \\
\text { grade IV }\end{array}$ & $9(40.9)$ \\
$\quad \begin{array}{l}\text { Anaplastic WHO grade III } \\
\text { Diffuse WHO grade II }\end{array}$ & $8(36.4)$ \\
Pilocytic WHO grade I & $2(09.1)$ \\
\hline
\end{tabular}

The tumour brain tissue and normal adjacent tissue of glioma were stained with two antibodies by usingCD133+/VEGFA+ markers to define angiogenic cell population that form new blood vessels and the co-localisation of the marker were shown in Fig 1. The angiogenic cells in brain tumour tissue were significantly higher compared to adjacent normal brain tissue (median $1.07 \pm 0.96 \%$ vs. median $0.69 \pm$ $0.68 \%$; The angiogenic cells in brain tumour tissue were significantly higher compared to adjacent normal brain tissue (median $1.07 \pm 0.96 \%$ vs. median $0.69 \pm 0.68 \%$; Wilcoxon signed rank test $Z=-3.100$; $\mathrm{p}=0.002$ ). Positive correlation was found between the angiogenic cells of brain tumour tissue and adjacent normal brain tissue (Spearman's rho correlation test, $r=0.56 ; p=0.007 ;$ Fig. 2 ).

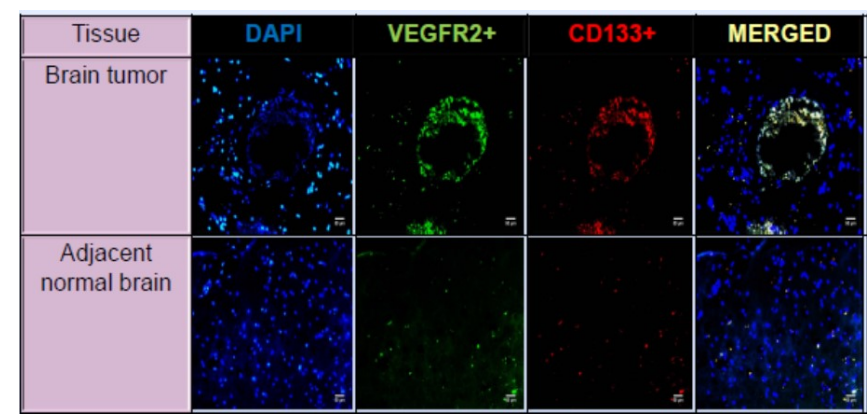

Figure 1. Immunofluorescence staining. The figure demonstrates the presence of tissue-resident angiogenic cells (CD133+/VEGFA+) in astrocytic glioma.

In circulating systems of astrocytic glioma patients we used CD133-/VEGFR2+ to define the matured endothelial cells. Stringent gating systems was applied in the flow cytometry analyses to get the percentage of PBMCs population that stained with CD133- and VEGFR2 + markers as shown in fig 3. Significant positive correlation was found between angiogenic cells in brain tumour and matured endothelial cells in circulating systems of astrocytic glioma patients (Pearson correlation test, $r=0.60, p$ =0.003; Fig 4). However no significant correlation was found between adjacent normal tissue brains and circulating matured endothelial cells (Pearson correlation test, $r=-0.022, p=0.923)$.

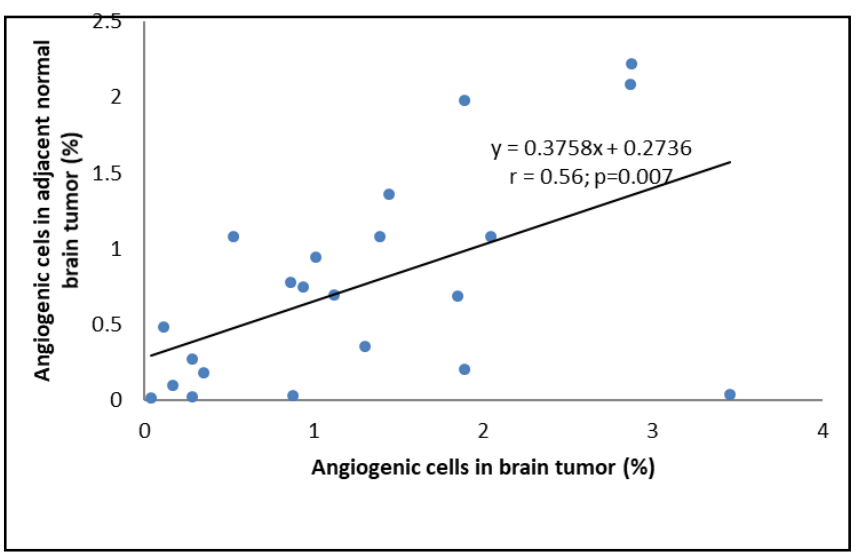

Figure 2. Correlation between angiogenic cells (CD133+/VEGFA+) in brain tumour and normal adjacent brain of astrocytic glioma patients.

\section{Discussion}

This study demonstrated the tumour tissue angiogenic cells that stained positive with CD133+/ 

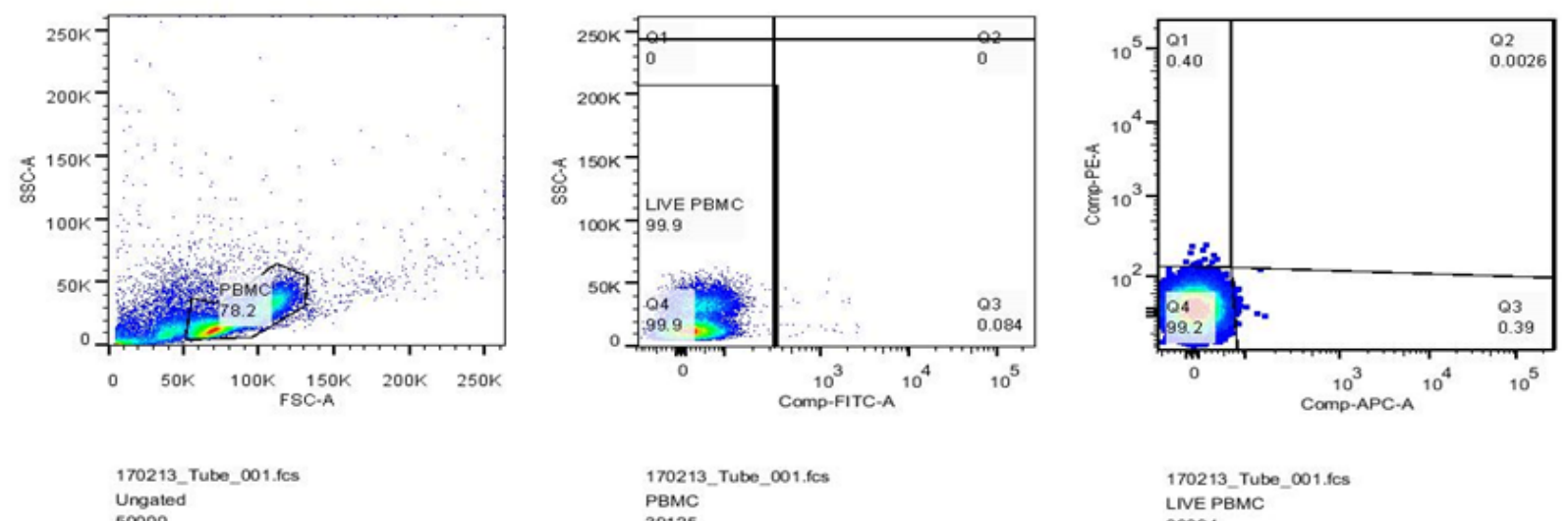

50000

170213_Tube_001.fcs

39125

170213_Tube_001.fcs

LIVE PBMC

39094
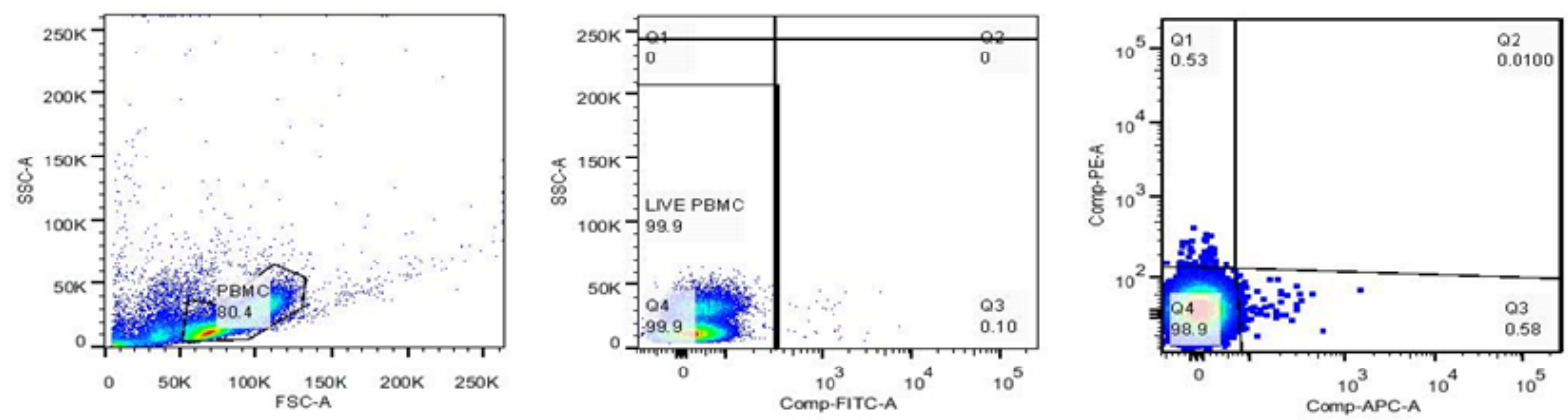

170213_Tube_002.fcs

Ungated

170213_Tube_002.fcs

PBMC
40178

170213_Tube_002.fcs

LIVE PBMC

40137

Fig 3. Flow cytometry showing the population of PBMCs and the cell population that stained with the markers

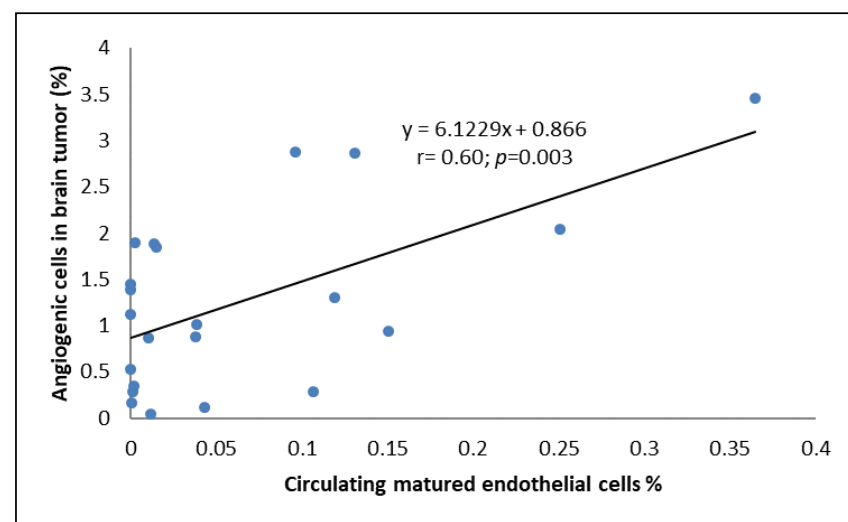

Figure 4. Correlation between angiogenic cells (CD133+/VEGFA+) in brain tumour and circulating matured endothelial cells (CD133-/VEGFR2+) of astrocytic glioma patients.

VEGFA+ markers have potential role in recruiting matured endothelial cells (CD133-/VEGFR2+) from the systemic circulation homing to vasculature for the tumour angiogenesis. The data showed correlation between the matured endothelial cells in the circulating and angiogenic cell population in the tumour. CD133+ cells exhibit the marker for immature endothelial progenitor cells. ${ }^{18}$ This suggest that the cells expressing VEGFR2+ that found on the surface of the endothelial cells and losing the expression of CD133+ marker because of differentiation of EPCs into matured endothelial cells. Therefore we suggest that the matured endothelial cells homing to the tumour tissue sites to enhance the tumour growth. This supported by the previous study that found the recruitment of bonemarrow derived endothelial progenitor cells to the site of tumour induced abnormal differentiation of these cells into matured endothelial cells which then incorporate into a subset of sprouting tumour neovessels. ${ }^{6-9}$

The VEGFR2 + expressed by glioblastoma multiforme as one of the lethal cancer with elevated VEGF and expressed on the cell surface of the CD133+ human glioma stem like cells. ${ }^{19}$ The bone marrow endothelial cells are single nucleus cells differentiated from EPCs and through luminal incorporation the formation of neovessels occurs at the tumour site. Significant reduction of EPCs has effects on impaired tumour growth and delay in the growth followed by reduction of tumour vessel density. Thus the EPCs have ability to differentiate into matured endothelial cells which plays important role in vessel formation and contribute both structurally and functionally to tumour neoangiogenesis. The previous study done by Daniel 
et al also defines the EPCs cells surface expression of VEcadherin,VEGFR2, CD31 low, Endoglin, and Prominin I/AC133 differentiate into matured endothelial cells. ${ }^{7}$ However the current study found no correlation with normal adjacent brain tumour and circulating matured endothelial cells. Therefore the circulating matured endothelial cells enhance the angiogenic activity at tumour site rather than adjacent normal brain in astrocytic glioma patients. The positive correlations exist between angiogenic cells in tumour and adjacent normal brain tissue suggesting that tumour angiogenic cells also recruited from the adjacent normal tissue angiogenic cells in astrocytic glioma.

CD133+ cells exhibit the marker for immature endothelial progenitor cells. ${ }^{18}$ The CD133+ cells coexpress together with VEGFA+ angiogenesis marker in the tumour tissues. The expression of CD133+/ VEGFA+marker indicate the CD133+ cells in the tumour tends to secrete the mitogen VEGFA+ to facilitate the recruitment of the endothelial cells from the circulating system. Therefore the data suggest that circulating matured endothelial cells homing towards thetumour site that still forming the new blood vessels from pre-existing vessels for the angiogenesis process. A study has been carried out on brain tumours and peripheral blood to access functional characteristics of CD133+ cells. The study demonstrated that the human brain tumours CD133+ cells are EPCs with angiogenic properties. The endothelial cells also involved in LDL uptake, vWF expression and tube formation in vitro compared with CD133- tumour cells. The CD133+ possesses in EPCs and has capability in formation of tumours with more vascularization. Furthermore the results showed that the mice implanted with CD133+ tumour cells showed bigger and more vascularized tumour compared to tumours made by $\mathrm{CD} 133^{-}$cell population. ${ }^{20}$

Other possibilities could be the growing tumour that may face hypoxia and blood vessel injury in the tumour as it proliferates and undergo differentiations of the cells and this might injure the existing blood vessels due to high shear pressure. This phenomenon eventually can induce the VEGFA+ secretion to repair the injured blood vessels in the tumour and then recruit the (CD133-/ VEGFR2+) matured endothelial cells from the circulating for the tumour angiogenesis process. The CD133+ also secretes the VEGF for the angiogenesis and tumour growth. Thus the angiogenesis plays a vital part in order to support the growth of the tumour in the astrocytic glioma patients. Whenever the vascular injury occurs, the circulating host derived endothelial cells will be highly demanded for the neoangiogenenesis processes. Similarly tumour growth angiogenic switch will lead to recruitment of EPCs from the bone marrow. ${ }^{7}$

Prominin-1/CD133 used as EPCs surface marker. The marker is classified as pentaspan transmembrane protein which regulates angiogenesis and apoptosis and effect vascular endothelial growth factor (VEGF). In a study of melanoma cell lines, the knocking down of prominin-1 in the cells induce VEGF binding reduction and more apoptosis. While in endothelial cell lines it causes disorganized and dysfunctional blood vessels as a result of knocking down of prominin-1 in the cells. In vitro analysis found that soluble prominin-1's interaction with VEGF is specific to VEGF164 which have the heparin binding domain increased VEGF164 dimer formation which stimulates VEGF164 binding to its receptors. ${ }^{21}$ This support the current findings that indicates the CD133+ cells coexpressing with VEGFA+ markers in tumour tissue correlated with CD133-/VEGFR2+ cells in the circulating systems. The expression of CD133+/VEGFA+angiogenic marker in the tumour indicate the CD133+ cells in the tumour tends to secrete the mitogen VEGFA+ to facilitate the recruitment of the matured endothelial cells (CD133 -/VEGFR2+) from circulating systems.

Disorganized vascular structures with aberrant permeability and diffuse leakage of perfused lectin solution into the stroma were observed in primary human dermal microvascular endothelial prominin-1 knocked down cells. Significant results were found in human dermal microvascular endothelial prominin -1 knocked down cells with $40 \%$ decrease in the total interconnected capillary structures length compared to control cells. The study also found that Prominin-1 interacts with VEGF on endothelial cells since no immunoprecipitation of either VEGF or prominin-1 resulted in pull down of the other protein were observed and further supported by co-localization of these two markers in immunocytochemistry as seen in confocal microscopy. Prominin-1 may hold significant potential as a target of cancer therapy since Prominin-1 expression enhance the proliferation, angiogenesis and cell survival with direct 
correlation. The study found that prominin-1 is essential for the growth of endothelial cells and melanoma cells while VEGF has properties of prosurvival and angiogenic characteristics in endothelial cells and it inhibits apoptosis proteins in cancer cells. ${ }^{21}$

Vascular endothelial growth factor (VEGF) that produced by primary tumour are responsible for the tumour proliferation and migration. The angiogenesis and mitogenic signal induced by VEGF binding to the VEGF receptors that found on the endothelial cells. Previous study have introduced radiation induced VEGF in cultured medium from irradiated glioma cells and the result showed strong motility of the tumour because the VEGF phosphorylate VEGFR2 causing the VEGFR2-mediate downstream signaling by activation of SrC and FAK phosphorylation that responsible for the migration and cell invasion in the glioblastoma multiformae tumour cells. ${ }^{22}$ The statement further supported by current result that showed the VEGFA+ expressing tumour associated with circulating VEGFR2+ cells in the study. The blocking of this action of radiation induced VEGF by anti-VEGF antibodies showed low tumour migration and cell invasion. The significant higher secretion of VEGF from media of irradiated glioma cells lines compared to unirradiated glioma cells was due to VEGF transcription. Therefore the glioma cells that found to be scattered surrounding a gross tumour area can be affected by the radiation therapy leaving the VEGF to be induced for the angiongenesis or tumour relapse that often happens during or after the glioblastoma multiformae treatment. Thus the combination of radiation therapy with anti-VEGF agent are necessary for better treatment. ${ }^{22}$

\section{CONCLUSIONS}

The findings of the study support the possible roles of EPCs in astrocytic glioma patients. Thus aiming the angiogenesis-targeted therapy in the astrocytic gioma will be beneficial to eradicate the tumour growth.

\section{ACKNOWLEDGEMENT}

The study is supported by Universiti Sains Malaysia short term grant 304/PPSK/61312016. Priscilla Das is a holder of MyBrain15-MyPhd scholarship.

\section{REFERENCES}

1. Schlag P. M and Senn, H.J. Gliomas. Recent Results in Cancer Research. Springer-Verlag Berlin Heidelberg, 2009. p. 1-259. DOI: 10.1007/978-3-540-31206-2.

2. Okada, H., Kohanbash, G., Zhu, X., Kastenhuber, E.R., Hoji, A., Ueda, R and Fujita, M. Immunotherapeutic approaches for glioma. Critical Reviews in Immunology 2009. 29(1): p. 1-42.

3. Janis B and Arbab, A.S. The role and therapeutic potential of endothelial progenitor cells in tumor vascularization. Scientific World Journal 2010. 10: p. 1088-1099.

4. Hillen, F and Griffioen, A.W. Tumour vascularization: sprouting angiogenesis and beyond. Cancer Metastasis Rev, 2007. 26(3-4): p. 489-502.

5. Döme, B., Hendrix, M.J., Paku, S., Tovari, J and Tímar, J. Alternative vascularistion mechanisms in cancer: pathology and therapeutic implications. Am J Pathol 2007. 170((1)): p. 115.

6. Benedetta, B., Deregibus, M.C.D and Camussi G. Characterization of molecular and functional alterations of tumor endothelial cells to design anti-angiogenic strategies. Current vascular pharmacology, 2010 (doi:10.2174/157016110790887036).

7. Nolan, D. J., Ciarrocchi, A., Mellick, A. S., Jaggi, J. S., Bambino, K., Gupta, S., et al.Bone marrow-derived endothelial progenitor cells are a major determinant of nascent tumor neovascularization. Genes Dev, 2007. 21: p. 1546-1558.

8. Dome, B., Dobos, J., Tovari, J., Paku, S., Kovacs, G., Ostoros, G and Timar, J. Circulating bone marrow-derived endothelial progenitor cells: Characterization, mobilization, and therapeutic considerations in malignant disease. Cytometry Part A, 2008. 73A (3): p. 186-193.

9. Charalambous, C., Chen, T.C and Hofman, F.M. Characteristics of tumor-associated endothelial cells derived from glioblastoma multiforme. Neurosurgical Focus, 2006. 20(4): p. 1-5.

10. Chi, A. S., Sorensen, A. G., Jain, R. K., and Batchelor, T. T. (2009). Angiogenesis as a Therapeutic Target in Malignant Gliomas. The Oncologist, 14(6), 621-636. http:// doi.org/10.1634/theoncologist.2008-0272 
11. Rafat, N., Beck G,Ch., Schulte,

J., Tuettenberg, J and Vajkoczy, P. Circulating endothelial progenitor cells in malignant gliomas. J Neurosurg 2010. 12: p. 43-49. .

12. Greenfield, J.P., Jin, D.K., Young, L.M, Christos, P.J, Abrey, L., Rafii, S and Gutin, P.H. Surrogate markers predict angiogenic potential and survival in patients with glioblastoma multiforme. Neurosurgery, (10): p. 2009 May;64(5):819-26; discussion 8267.

13. Ahluwalia, M.S and Gladson C.L. Progress on Antiangiogenic Therapy for Patients with Malignant Glioma. Journal of Oncology, 2010. 2010.

14. Laperriere, N., Zuraw, L., and Cairncross, G. Radiotherapy for newly diagnosed malignant glioma in adults: a systematic review. Radiother Oncol, 2002. 64(3): p. 259-73.

15. Laws E.R., Parney I.F., Huang W., Anderson F., Morris AM., Asher A., Lillehei KO., Bernstein M., Brem H., Sloan A., Berger, M.S and Chang, S. Glioma Outcomes Investigators. Survival following surgery and prognostic factors for recently diagnosed malignant glioma: data from the Glioma Outcomes Project. J Neurosurg, 2003. 99(3): p. 467-73.

16. Parney, I.F., Waldron J.S., and Parsa A.T. Flow cytometry and in vitro analysis of human glioma-associated macrophages. Laboratory investigation. J Neurosurg, 2009. 110(3): p. 572-82.

17. Fava, G., Ruini C., and Rafanelli C. Psychometric Theory Is an Obstacle to the Progress of Clinical Research. Psychother Psychosom, 2004. 73: p. 145 - 148.

18. Thomas J. P., Katherine L. Z., Enrikas V., Jennifer F. Kherani., Pascal J. GoldschmidtClermont and Eric D. Peterson. Common endothelial progenitor cell assays identify discrete endothelial progenitor cell populations. American Heart Journal, 2009. 157(2): p. 335-344.

19. Hamerlik, P., Lathia, JD., Rasmussen, R., Wu ,Q., Bartkova, J., Lee, M., Moudry, P., Bartek, J. Jr. , Fischer, W., Lukas J, Rich, $\mathrm{J} \mathrm{N}$ and Bartek, J. Autocrine VEGF-VEGFR2Neuropilin-1 signaling promotes glioma stemlike cell viability and tumor growth. The Journal of Experimental Medicine, 2012. 209 (3): p. 507-520.

20. Choi, S.A., Wang, K.C., Phi, J.H., Lee,
J.Y., Park., C.K., Park, S.H and Kim, S.K. A distinct subpopulation within CD133 positive brain tumor cells shares characteristics with endothelial progenitor cells. Cancer Letters, 2012. 324(2): p. 221-230.

21. Adini A., Adini I., Ghosh K., Benny O., Pravda E., Hu R., Luyindula D and D'Amato RJ. The stem cell marker prominin-1/CD133 interacts with vascular endothelial growth factor and potentiates its action. Angiogenesis, 2013. 16 (2): p. 405-416.

22. Kil, W., Tofilon. P., and Camphausen K. Postradiation increase in VEGF enhances glioma cell motility in vitro. Radiation Oncology, 2012. 7(1): p. 25. 